\title{
Cranial Functional (Psychogenic) Movement Disorders
}

Kaski D* ${ }^{* 1,2}$ MBBS PhD, Bronstein AM ${ }^{1,2}$ FRCP PhD, Edwards $\mathrm{MJ}^{1,4}$ MBBS PhD,

Stone $\mathrm{J}^{+3} \mathrm{FRCP} \mathrm{PhD}$

Affiliations:

${ }^{1}$ Department of Neuro-otology

National Hospital for Neurology and Neurosurgery

Queen Square

London

United Kingdom

004402033135526

d.kaski@imperial.ac.uk

* corresponding author

${ }^{2}$ Division of Brain Sciences

Imperial College London

Charing Cross Hospital

London

United Kingdom

${ }^{3}$ Department of Clinical Neurosciences

University of Edinburgh

Western General Hospital

Edinburgh

${ }^{4}$ Sobell Department of Motor Neuroscience and Movement

UCL Institute of Neurology

London

United Kingdom

\section{Word count:}

Title: 8

Main text: 3646

Abstract: 93

Figures: 5

Videos: 10

Running head: Cranial functional movement disorders 


\section{Author contributions:}

DK compiled the manuscript, created the figures, and edited the videos. JS compiled the manuscript, created figures, provided patient videos, and approved its final version. AMB provided patient videos, compiled the manuscript and approved its final version and MJE compiled the manuscript and approved its final version.

AMB is supported by a research grant from the Medical Research Council (UK). JS is supported by an NRS Career Fellowship from NHS Research Scotland.

The authors report no conflicts of interest. 


\begin{abstract}
Functional (psychogenic) neurological symptoms are commonly encountered in neurological practice. Cranial movement disorders - i.e. affecting the eyes, face, jaw, tongue, or palate - are an under-recognized feature of patients with functional symptoms. They may present in isolation, in the context of multiple functional symptoms, or be found only on examination. Whilst the field of functional neurological disorders has expanded, an appreciation of cranial functional movement disorders is still lacking. Moreover, identification of the positive features of functional cranial movement disorders such as convergence and unilateral platysmal spasm may lend diagnostic weight to a suspected functional neurological disorder. In addition, around $10 \%$ of patients with stroke mimics have functional disorders. Thus, an appreciation of functional cranial disorders is particularly timely with advances in stroke therapy, where there is potential physical and psychological harm in misdiagnosis associated with thrombolysis treatment.
\end{abstract}

Keywords: Eye movements; Facial movements; Psychogenic; Functional; Movement disorders. 


\section{Introduction}

Cranial movement disorders are movement disorders that affect the eyes, face, jaw, tongue or palate. Cranial movement abnormalities are perhaps an under-recognized feature of patients with functional (psychogenic) neurological symptoms ${ }^{1-3}$, and in our experience a common concomitant of other functional neurological symptoms. Between 5 and $50 \%$ of all patients with functional movement disorders present with a facial movement disorder ${ }^{3}$ but the prevalence of functional eye movement disorders is largely unknown. Patients may present overtly with a complaint suggestive of an eye movement disorder (e.g. double vision) or a facial symptom (e.g. 'drooping mouth') with or without additional neurological symptoms. More rarely, in a patient with functional neurological symptoms, functional eye movement abnormalities and less commonly facial abnormalities are found only on formal examination where they can cause diagnostic confusion. As with other motor disturbances such as weakness or tremor, such phenomena are particularly amenable to objective clinical assessment (in contrast to subjective report of sensory symptoms), and therefore provide an excellent opportunity to make a positive and specific diagnosis of a functional movement disorder.

The field of functional neurological disorders has expanded, with increasing awareness that the diagnosis and treatment of these disorders is a neurologist's responsibility. Nevertheless, functional cranial movement disorders are still relatively neglected in the literature ${ }^{1}$. Failure to correctly classify and diagnose functional disorders may lead to significant iatrogenic damage, and deny the patient the appropriate treatment. It is not uncommon for patients to be sent from one specialist to another with a variety of symptoms not explained by multiple laboratory or 
radiological investigations. A positive diagnosis of a functional disorder can break this cycle and lead to physical or psychological rehabilitation. Moreover, accurate diagnosis has particular implications for the diagnosis of acute stroke, where inappropriate treatment with thrombolysis could have detrimental consequences. Finally, the lack of treatment studies for cranial functional movement disorders underpins the need for increased awareness and improved study of these disorders, given the potential for phenotype-specific treatment outcomes ${ }^{1}$.

In this review we highlight those facial and ocular symptoms that can have a functional origin, and review the common symptoms and positive clinical signs of functional cranial movement disorders and contrast these with some key signs of organic pathology to aid diagnosis and a rational approach to management. We discuss all those cranial functional movement disorders that we have come across in general neurology, specialist movement disorder and balance and eye movement clinical practice. Finally, we discuss the current state of the field and provide our thoughts on the direction for future research.

Whilst we recognize that there are pros and cons to the terms "functional" and "psychogenic" 4,5 , in this manuscript we will refer to these conditions as functional.

\section{Functional Eye Movement Disorders}

\section{Convergence Spasm}

Convergence is a normal eye movement reflex occurring when gaze is shifted from a distant object to a near object. Convergence spasm refers to the abnormal persistence of this movement when the patient is not fixating on a near object (Fig. 1). 
Convergence spasm is the most commonly reported functional eye movement disorder, occurring in as many as $69 \%$ of functional movement disorder cases in one small study of 13 patients ${ }^{2}$. Patients with convergence spasm may complain of blurry vision when looking at a distance following near fixation (e.g. reading). Other symptoms include intermittent blurred vision that typically corrects by squeezing eyelids tight shut and opening them again, and intermittent diplopia. Symptoms usually last seconds but may be described as continuous when one episode of spasm is succeeded by another.

Convergence spasm is demonstrated when the patient is asked to look at a near object $(30-40 \mathrm{~cm})$, sometimes the doctor her/himself. It is often triggered by formal ocular examination whereby the patient's angle of convergence becomes clearly too large for the visual target offered and the pupils become excessively miotic. On moving the target back away or on asking the patient to look at a distant object (the wall behind the examiner) the convergence and miosis persist. If the fixation target is then moved to lateral gaze the excessive convergence also continues - Videos $1 \& 2$ show how, during lateral gaze, one or both eyes remain adducted with strong medial rectus contraction. As a consequence patients with convergence spasm are often misdiagnosed as having unilateral or bilateral abducens nerve palsies ${ }^{6}$. Formal oculographic recordings may facilitate the diagnosis (Fig. 2B). Convergence spasm can be differentiated from an abducens palsy ${ }^{7}$ by:

a) presence of miosis in convergence spasm;

b) full range of movements demonstrated by rapid, small amplitude passive head turns (head impulse test ${ }^{8}$ ), optokinetic stimuli, or Dolls-eye manoeuvre; 
c) absence of other oculomotor signs, such as gaze paretic nystagmus (nystagmus that occurs in the adducting [normal] eye);

d) appearance of the convergence spasm only during formal examination but not during casual observation during history taking.

Organic causes of convergence spasm, also termed spasm of the near reflex, are rare and include disease at the diencephalic-mesencephalic junction (thalamic esotropia), Wernicke-Korsakoff syndrome, posterior fossa lesions, epilepsy, and phenytoin toxicity ${ }^{7}$. In many of these reported cases however the description of the symptoms and signs actually suggest convergence spasm comorbid to the underlying organic problem, or in the case of Wernicke's perhaps bilateral abducens nerve palsies.

Finally, convergence spasm has been reported in patients with benign paroxysmal positional vertigo, during provocation (positional) manoeuvres ${ }^{9,}{ }^{10}$, or mimicking benign paroxysmal positional vertigo ${ }^{22}$, findings that we have also observed. A case of spontaneous convergence spasm (un-related to positional manoeuvres) has also been reported in the context of a peripheral vestibulopathy ${ }^{11}$. Such cases may relate to a voluntary attempt to suppress the nystagmus (and thus reduce symptoms), or a functional response to disabling vertigo.

Caution is required in over-interpreting mild convergence spasm. For example convergence spasm was found not only in 4/11 patients with an organic movement disorder but also in $4 / 12$ healthy controls ${ }^{2}$. This finding is rather surprising given how rare it is in our experience to find convergence spasm in healthy subjects in neuro-otology and eye movement clinics. One must bear in mind that 
convergence/divergence are under voluntary and reflex oculomotor control, and are thus the only dysconjugate ocular movement that can be initiated voluntarily .

\section{Convergence Paralysis}

Convergence insufficiency or paralysis describes a partial or complete failure of convergence. Here, diplopia exists only at near fixation, adduction is normal, and the patient is unable to converge ${ }^{12}$. Accommodation may be normal, reduced, or absent. Patients will often report difficulty reading, particularly at close range, and blurring of vision. Differentiating organic from a functional convergence paralysis is a greater clinical challenge than diagnosing convergence spasm because it represents an absence of movement rather than the generation of a complex oculomotor action.

In organic convergence paralysis, as in normal ageing or neuro-degeneration ${ }^{13}$, convergence will always be absent. In functional convergence paralysis, convergence movements may be observed during the "casual examination" when the patient is performing other near tasks such as looking at their own wristwatch ${ }^{14}$, or by asking the patient to read out his/her prescriptions. One other practical method of eliciting convergence in patients with suspected functional convergence paralysis is asking the patient to follow a visual target (e.g. the examiner's finger) from side to side, whilst subtly and slowly moving the target closer to the patient's nose.

\section{Gaze limitation (Video 3)}

Functional gaze limitation most often manifests on formal testing of eye movements rather than presenting as a primary complaint. Patients may have eyelid fluttering on attempted eye movements, and may have effortful facial movements or facial 
grimacing ${ }^{15}$ exclusively during the examination (Videos $3 \& 4$ ). Many patients will also report pain on eye movements, especially upgaze testing in patients with headache, with a tendency to avoid moving the eyes on the formal examination, but no apparent discomfort during 'casually observed' saccades. There may be inability to move the eyes in vertical ${ }^{16}$ or horizontal directions ${ }^{17}$ when patients are asked to follow a visual target, but with full ocular excursions during optokinetic stimuli or passive head rotations. Patients may complain of diplopia despite conjugate eye movements (i.e. a normal alignment of both eyes).

Functional gaze limitation may accompany symptoms such as poor mobility together with globally slow movements that may suggest Parkinsonism ${ }^{18}$. It can be distinguished from organic supranuclear palsies by the presence of normal saccades in the casual examination, normal pursuit, and the presence of variability. Most organic causes of supranuclear gaze palsy will be accompanied by slow saccades, which are not seen in functional disorders. Furthermore, the diagnosis of functional vertical gaze palsy can usually be aided by the finding that the eyebrows do not elevate during attempted upward gaze (whereas the eyebrows do elevate in organic vertical gaze palsy) ${ }^{16}$.

\section{Functional and voluntary nystagmus/oscillopsia}

The term "Voluntary Nystagmus" (VN) has been used to describe a high frequency, horizontal, low amplitude eye oscillation that can be voluntarily initiated and terminated ${ }^{19}$. Functional nystagmus refers to the same phenomenon but experienced as an involuntary symptom with oscillopsia (wobbly, unstable or blurred vision in 
which the images are 'jumping'). A survey by Zahn ${ }^{20}$ showed that about $8 \%$ of college students can produce voluntary nystagmus at will.

The eye oscillations of VN (Fig. $2 \mathrm{~B}$ and Videos $5 \& 6$ ) are conjugate and highfrequency. The term "nystagmus" is in fact incorrect as in $\mathrm{VN}$ and functional nystagmus the slow phase eye movement that characterises actual nystagmus (Fig. 2A) is absent. $\mathrm{VN}$ is confined to horizontal oscillations, and these may be superimposed on smooth pursuit movements, and may also be accompanied by a head tremor as well as eyelid flutter ${ }^{21}$. Typically, VN cannot be maintained for more than 25 seconds ${ }^{20}$ (although usually much briefer; Video 5) and the nystagmus tends to decrease in amplitude and duration during this period. Ocular flutter, the main differential diagnosis, is in contrast persistent and usually there are associated cerebellar or brainstem oculomotor, or pyramidal signs ${ }^{22}$.

Patients with functional nystagmus may present with oscillopsia. Like many of the functional disorders of the eyes and face, functional nystagmus may be triggered by the examination, especially of eye movements, but may not be present during a casual examination. There may be convergence at the onset of the nystagmus (Video 6), sometimes amounting to full convergence spasm, as well as diminution in the intensity and frequency of the nystagmus with repeated examination. A patient with functional nystagmus is described in Box 1.

Although the combination of intermittent oscillopsia and diplopia may suggest a combination of convergence spasm and functional nystagmus there are alternative 
explanations for this symptom complex (Table 1), thus a careful examination must always be carried out.

\section{A note on diplopia}

Diplopia may be a symptom of a functional eye movement disorder. Organic diplopia is typically binocular (disappears when one eye is covered) and is the result of dysconjugate gaze (a failure of the eyes to turn together in the same direction). Thus, there will be a limitation of movement of one or more ocular muscles, which may be confirmed clinically, or using a Hess chart. Asking the patient to look in the direction of the suspected weak muscle will increase the diplopia (e.g left lateral gaze for a left lateral rectus weakness) in organic diplopia. Monocular diplopia may point to ocular pathology (e.g. retinal disease, refractive errors, abnormalities of the cornea and lens), and more rarely visual cortex lesions ${ }^{23}$. In the presence of organic pathology one image is clear and the other is blurred ${ }^{24}$. Patients with a functional monocular diplopia will often be unable to identify whether one image is blurred or not. Thus, true monocular diplopia, where two separate and equal images of an object are seen with one eye only, almost always indicates a functional disorder. Triplopia and polyopia can also be functional although in a review of 13 cases of triplopia, 11 had an organic diagnosis ${ }^{25}$.

\section{Functional Upper Facial Movement Disorders}

The commonest type of functional movement disorder in the ocular region of the face is eye closure (Video 9). This typically presents with episodic and unilateral (though

can be bilateral, as in Video 9) contraction of orbicularis oculis ${ }^{3}$ (Fig. 3B). The 
orbicularis contraction may appear jerky and the muscular oscillations can sometimes be felt by gently placing a finger over the affected eyelid. Eye closure may be mistaken for ptosis, and so the term pseudoptosis has sometimes been used to describe this situation. Pseudoptosis is however misleading since eye closure is usually related to a contraction of orbicularis muscle around the eye rather than failure of levator palpabrae ${ }^{26}$, although we have seen the latter rarely. Alternatively, overcontraction of orbicularis oculis can mimic a unilateral weakness of frontalis with an apparent inability to elevate the eyebrow on the affected side, or an eyebrow that is 'depressed' relative to the normal side (Fig. 4C). When the patient attempts to elevate the eyebrow or look up this may increase contraction of orbicularis oculis in a functional facial movement disorder.

In organic hemifacial spasm (see next section) there may be elevation of the eyebrow on the same side as eye closure resulting from co-contraction of orbicularis oculi and the internal part of frontalis (Fig. 4B). This is sometimes called "the other Babinski sign" 27,28 and helps differentiate organic hemifacial spasm from functional orbicularis oculis contraction in which there is sometimes spontaneous overactivity of the contralateral frontalis ${ }^{3}$ (Fig. 4C). Interestingly, "the other Babinski sign" has never been reported in blepharospasm (a focal dystonia characterized by forceful, involuntary, spasmodic contraction of the orbicularis oculi; Fig. 4A). Neurophysiological studies assessing the blink reflex may also be helpful to disambiguate between essential and presumed functional blepharospasm ${ }^{29}$. In organic blepharospasm the blink reflex recovery cycle is abnormal as a result of abnormal brainstem interneuron excitability, whereas in functional eye closure the blink reflex is normal. Resolution of symptoms during specific tasks will be of diagnostic help; 
one patient of ours had bilateral complete eye closure that resolved when he was playing video games.

\section{Functional Movement Disorders of Mouth, Tongue and Palate}

Several distinctive features point towards a functional movement disorder of the mouth, tongue, or palate. Functional hemifacial spasm is seen in $2-6 \%$ of patients referred for specialist evaluation of hemifacial abnormal movements ${ }^{30}$, although more data is required to establish the true frequency. In a landmark case series by Fasano et al, most described patients were female (92\%) and relatively young (mean age $37 \mathrm{yrs})^{3}$. Platysma is commonly contracted which leads to the corner of the mouth being pulled down on one side giving an appearance of weakness (Video 8). Less commonly, the corner of the mouth may be elevated compared to the other side (Videos $9 \& 10$ ). Jaw deviation to the affected side is a common and specific sign in many patients (Video 7). In many patients with functional facial spam, there is evidence of a functional motor and sensory disorder in the ipsilateral arm and leg ${ }^{2}$. Of note, the presence of motor symptoms in the upper and lower halves of the face and the ipsilateral arm and leg usually requires more than one neurological lesion to have an anatomical basis - a fact that may be useful to help both patients and nonneurologists understand the rationale for a functional diagnosis. The combination of platysmal spasm leading to the appearance of 'mouth drooping', and hemiparesis understandably can lead to concerns about acute stroke, especially health professionals uncritically applying the FAST- face, arm, leg, time - test for acute stroke (Fig. 3). Functional disorders account for around $10 \%$ of stroke mimics in published series ${ }^{31}$. 
Functional movement disorders affecting the tongue typically co-exist with functional hemifacial spasm. In functional hemilingual spasm the tongue usually deviates towards the side of a facial spasm (i.e. the spasm is all on the same side; Fig. 5C). In an organic hypoglossal palsy, if the lesion is supranuclear (e.g. following a stroke) only the lower half of the face should be weak and the tongue also deviates towards the same side as the affected part of the face (and away from the lesion) (Fig. 5A) ${ }^{32}$. If the lesion is in the pons/medulla then the ipsilateral face (including eye) may be weak (from seventh nerve involvement), the tongue will deviate towards the lesion, towards the affected half of the face but away from the side of limb weakness (Fig. 5B). The differential diagnosis of facial hyperkinetic movement disorder ${ }^{33}$ is wide, and making the diagnosis requires familiarity with these entities. Table 2 indicates the commonest causes and the clinical features that help distinguish them from functional facial spasm. Most of these diagnoses involve briefer movements in facial muscles rather than the sustained contractions typically seen in patients with a functional disorder.

Palatal tremor (often termed palatal myoclonus) can also occur as a functional movement disorder ${ }^{34,35}$. In a retrospective description of 17 patients with isolated palatal tremor 10 patients were deemed to have functional palatal tremor on the basis that the tremor was variable (spontaneous changes in frequency, amplitude, direction, or characteristics), entrainable (brought into a specific rhythm or frequency by asking the patient to copy a motor activity of a given frequency), and distractible (a change in tremor amplitude, direction or quality, with a decrease or cessation of tremor, when volitionally performing other cognitive or motor tasks) ${ }^{36}$. For example, palatal tremor 
transiently stopped when patients were asked to tap with their fingers to a rhythm set by the examiner or to perform ballistic reaching movements with the arms ${ }^{36}$. Such patients are mostly female and younger (mean age at onset 35 versus 54 years) than patients with organic palatal tremor ${ }^{36}$, and frequently have a physical precipitating event such as a sore throat. These patients were more likely to have functional rocking movements of the head or neck, functional facial spasms, or functional visual disturbance. Ear clicking is particularly common in people with functional palatal tremor in our experience. Organic palatal tremor may be associated with pendular nystagmus, myoclonus, and cerebellar signs, and there may be an abnormality of the inferior olive on magnetic resonance imaging ${ }^{37,38}$.

\section{Generalised facial hyperkinetic or hypokinetic disorders}

Tics can sometimes be part of functional movement disorder and will sometimes affect the face and eyes. In a series of nine patients with functional/psychogenic tics eight of the patients had hyperkinetic facial movements. The authors of this paper suggest several clinical features can help differentiate functional from organic tics ${ }^{39}$; patients with functional tics tend to have an older age of onset (mean age 29.7yrs), a lack of premonitory sensation, an inability to transiently suppress the movements, a lack of family history of Tourette syndrome ${ }^{40}$, a lack of response to dopamine receptor antagonists, and the coexistence of associated functional movement disorder elsewhere in the body, such as limb tremor or weakness or non-epileptic seizures ${ }^{41}$.

Just as limitation of eye movement may occur as a functional movement disorder, some patients present with weakness or inability to move facial muscles, which may 
be accompanied by an inability to speak (aphonia). In our experience such patients have hypofunctioning of the vocal cords, whereby the cords approximate but remain open during attempted phonation ${ }^{42}$ (but patients have a normal cough). Patients in this situation are sometimes thought to have facial hypomimia as seen in Parkinson's disease and other parkinsonian disorders ${ }^{18}$. Such apparent muscle weakness or slowness is typically variable and distractible, particularly during the history taking.

\section{Common features of examination findings}

Many paroxysmal functional movement disorders (particularly of the eyes) will be present during the consultation, whereas it is usually very difficult to trigger organic paroxysmal eye movement disorders in the clinic (such as superior oblique myokymia or vestibular paroxysmia; Table 1). In a functional cranial movement disorder the movements may be induced by sustained contraction of facial or ocular muscles (e.g. longer than 10 seconds) ${ }^{3}$. As voluntary facial movements may also exacerbate synkinetic movements seen after facial palsy and sometimes in hemifacial spasm, it is the finding of sustained orbicularis oculis or platysma contraction that is the key finding. In some patients, examination of eye movements (Video 10) or a light stimulus (using a pen torch) may trigger the abnormal movements, which more clearly indicates a functional disorder (Video 11 and see also Fig. 3D and Box 2).

\section{Pathophysiology}

The individual vulnerability to specific cranial functional movement disorders and the selective expression of these (i.e. why it may affect the eyes in one patient, and the tongue in another) are largely unanswered questions that may have important therapeutic implications. Although the pathophysiology of cranial functional 
movement disorders is likely no different to other functional movement disorders, one reason why patients may manifest a cranial functional movement disorder, over for example a functional limb tremor, may be the co-existence of organic pathology in the face or eyes, prior injury to these areas, or an increased focus of attention on these areas by family members or doctors (e.g. functional nystagmus - see also Box 1). Thus, enquiring about associated physical events and symptoms found in some patients that include dental work, migraine and injury to the face that may help explain why the symptom began in the face and not somewhere else ${ }^{43}$. On a related note, the presence of a functional disorder should always be a 'red flag' to the clinician that there may be an additional disease process which has triggered the abnormality and which may need to be identified ${ }^{44}$.

Neuroimaging findings in patients with functional movement disorders have shown alterations in brain circuits mediating, amongst other things, awareness (e.g. anterior cingulate cortex, insula, amygdala) and self-referential processing (posterior parietal cortex, temporoparietal junction) ${ }^{45}$. Indeed, neurophysiology and neuroimaging offer potential insights regarding the mechanism of functional movement disorders ${ }^{46-48}$, but apart from the blink reflex cycle ${ }^{29}$ these have not yet been specifically applied to cranial functional movement disorders.

\section{Treatment options}

General concepts

Treatment options for cranial functional movement disorders are based on case series, case reports, and expert opinion consensus rather than randomised controlled trials, 
although much better treatment evidence is now emerging for functional motor disorders ${ }^{49-52}$ and non-epileptic seizures ${ }^{53-55}$.

Treatment begins with a positive clinical diagnosis and clear explanation of the nature of the problem, including giving a diagnostic label to the patient ${ }^{56}$ (observational study). Motor signs can form an important part of the explanation of the diagnosis to the patients, such as using pictures, videos or eye movement recordings to explain how limitation of gaze during eye movement examination disappears when a patient is distracted ${ }^{57}$ (observational study). This also emphasises the potential for reversibility and that the problem is 'software, not hardware'. This process of "rational persuasion" can set the scene for a therapeutic discussion about functional disorders; that they are common, genuine, but different from neurological disease, and potentially reversible and that they have positive clinical signs that have enabled the diagnosis to be made. This step alone can produce major therapeutic benefit and may in some patients be the only treatment necessary, perhaps by altering fundamental beliefs that are driving abnormally focused attention towards the symptom ${ }^{58}$. This step is not about 'reassurance' that there is "nothing wrong" but rather about providing an explanation of 'what is wrong' that reduces abnormal self-focused attention. For example, we sometimes encounter teenagers with brief paroxysmal oscillopsia (functional nystagmus) in the context of 'tired eyes', lack of sleep and anxiety when revising for university admission exams. Anxiety from parents and doctors alike, and unnecessary (normal) investigations tend to increase the frequency of the attacks. The attacks can however be reduced or aborted with a convincing explanation, and consequent reduced focus on the problem (see Box 1). 
There is little evidence for the use of drug therapy in the treatment of functional movement disorders at the present time, though medical treatment of associated neurological problems (e.g. migraine or anxiety/depression) is sometimes needed. Our personal clinical experience is that the prognosis of functional eye and facial movement disorder is generally good, although not universally so, where facilities exist to provide patients with a thorough explanation of the cause of the symptoms, and engage in therapy with appropriate follow-up, as for other extra-cranial functional movement disorders ${ }^{49,50}$.

Research on functional cranial movement disorder requires better epidemiological studies to help understand the scale of the problem, improved diagnostic accuracy and better use of digital technology to allow patients both to capture intermittent eye movement problems and potentially as a form of biofeedback for treatment.

\section{Functional eye movement disorders}

For functional eye movement disorders, our own experience indicates that symptomatic improvement is aided by explaining that the problem is genuine or visible, relates to overactivity or underactivity in oculomotor pathways, that those pathways are not damaged, and that eye movements can be normalised through eye movement retraining. This can inform discussions about whether further investigations are needed and provide a logical basis for treatment approaches, especially those that are designed to retrain the nervous system. Thus exercises from an optometrist (e.g. asking the patient to make eye movements between targets that are gradually moved further apart in patients with functional limitation of gaze) may be helpful in some patients if they know that the purpose is to relax a movement 
pathway that has become overactive. Symptomatic benefit in convergence spasm has also been reported in small patient groups with cycloplegic agents (e.g. atropine drops) that cause temporary paralysis of the cilliary muscles in combination with reading glasses ${ }^{59}$, and miotic agents used as placebo ${ }^{60,61}$ although the evidence for functional eye movement disorders is anecdotal (observational studies).

\section{Functional facial movement disorders}

In a study of 61 patients with functional facial movement disorder various treatments including psychotherapy and pharmacotherapy (including botulinum toxin) resulted in improvement in only $20 \%$ and another $20 \%$ remitted spontaneously ${ }^{3}$. Botulinum toxin in the context of facial movement disorders (observational study) may help some patients appreciate the reversibility of the problem, but symptoms may recur and therefore suggest the need for ongoing treatment, and so its role in this situation is uncertain.

For patients with episodically overactive facial muscles, learning how to trigger the movements can be helpful in treatment. So for example using prolonged facial muscle contraction, or removal of sunglasses if there is photophobia, affords an opportunity to experiment with the use of contrary strategies such as relaxation techniques (of facial muscles), distraction techniques, and graded deconditioning (e.g. the use of graded light sources in patients with light-induced eyelid closure) to try to overcome the involuntary movements in a more controlled way (observational studies). The use of deconditioning, accompanied by management of anxiety (including anticipatory anxiety) and relaxation techniques may also be of benefit (observational studies). 
There have been recent reports of transcranial magnetic stimulation techniques for the treatment of functional movement disorders ${ }^{62-67}$. Some case series do suggest benefit but it is not possible to separate the effects of TMS in these studies from other generic treatment approaches described above.

\section{Conclusions and future directions}

Functional cranial movement disorders are diagnosed, like functional motor disorders, in relation to their response to altered attention and distraction, either involving another movement or during the "casual" examination when the patient's attention is focused on something else. We have also highlighted how abnormal cranial movements (especially of the eye) may be triggered by the act of formal neurological examination when the patient is asked to attend excessively to automatic actions (e.g. saccadic eye movements to command). This is consistent with the idea that functional movement disorders are at one level disorders of abnormally focused attention, driven by expectations and beliefs regarding motor and sensory function.

One particular challenge is to investigate the dividing line between what makes a symptom voluntary and involuntary. Symptoms that can be reproduced by some healthy subjects voluntarily such as nystagmus create a model to do that. Thus, comparing movement-related cortical potentials (Bereitschaftspotential) that typically occur $1 \mathrm{~s}$ to $2 \mathrm{~s}$ prior to the electromyogram activity in subjects with voluntary nystagmus and patients with functional nystagmus may help determine whether a common (voluntary) mechanism is implicated in both, or not. It is important to state that this mechanistic approach to the study of functional disorders does not negate an 
important role, in some, even many, patients for psychosocial factors in conferring vulnerability to developing such symptoms and also in their triggering and maintenance. A collaborative approach between neurological and psychological approaches to research and development of clinical services is essential. Much work is still required to define optimal treatment regimes, with both general treatment strategies and tailored individual therapies, and a need for well-designed randomized controlled trials.

\section{Selection strategy and search criteria}

We identified articles with PubMed and Google Scholar searches for combinations of "non-organic" or "psychogenic" or "psychosomatic" or "somatoform" or "hysteria" and "ocular" or "eyes" or "accommodation" or "near reflex" or "facial" or "palate" or "tongue". We reviewed articles published from 1950 to July 2015, but focused on those articles published after 2004. We identified further articles by reviewing reference lists within other articles, irrespective of year of publication. Prospective, randomised, controlled studies were absent so methodological limitations are discussed. Primary references cited within textbooks, when available, were also reviewed irrespective of date of publication. Only publications written in French, German, or English were reviewed.

\section{References}

1. Espay AJ, Lang AE. Phenotype-specific diagnosis of functional (psychogenic) movement disorders. Current neurology and neuroscience reports. 2015; 15(6): 32.

2. Fekete R, Baizabal-Carvallo JF, Ha AD, Davidson A, Jankovic J. Convergence spasm in conversion disorders: prevalence in psychogenic and 
other movement disorders compared with controls. J Neurol Neurosurg Psychiatry. 2012; 83(2): 202-4.

3. Fasano A, Valadas A, Bhatia KP, Prashanth LK, Lang AE, Munhoz RP, et al. Psychogenic facial movement disorders: clinical features and associated conditions. Mov Disord. 2012; 27(12): 1544-51.

4. Fahn S, Olanow CW. "Psychogenic movement disorders": they are what they are. Mov Disord. 2014; 29(7): 853-6.

5. Edwards MJ, Stone J, Lang AE. From psychogenic movement disorder to functional movement disorder: it's time to change the name. Mov Disord. 2014; 29(7): 849-52.

6. Faucher C, De Guise D. Spasm of the near reflex triggered by disruption of normal binocular vision. Optom Vis Sci. 2004; 81(3): 178-81.

7. Leigh RJZ, D.S. The neurology of eye movements. 3 ed. New York: Oxford University Press; 2006.

8. Halmagyi GM, Curthoys IS. A clinical sign of canal paresis. Arch Neurol. 1988; 45(7): 737-9.

9. Gordon CR, Almog Y. Positional convergence spasm mimicking benign paroxysmal positional vertigo. Neurology. 2012; 78(9): 681-2.

10. Zappia JJ. Benign paroxysmal positional vertigo. Curr Opin Otolaryngol Head Neck Surg. 2013; 21(5): 480-6.

11. Dagi LR, Chrousos GA, Cogan DC. Spasm of the near reflex associated with organic disease. Am J Ophthalmol. 1987; 103(4): 582-5.

12. Bradley WGE. Neurology in clinical practice: Butterworths; 1990.

13. Biousse V, Skibell BC, Watts RL, Loupe DN, Drews-Botsch C, Newman NJ. Ophthalmologic features of Parkinson's disease. Neurology. 2004; 62(2): 177-80.

14. Keane JR. Neuro-ophthalmic signs and symptoms of hysteria. Neurology. 1982; 32(7): 757-62.

15. Thenganatt MA, Jankovic J. Psychogenic tremor: a video guide to its distinguishing features. Tremor Other Hyperkinet Mov (N Y). 2014; 4: 253.

16. Bruno E, Mostile G, Dibilio V, Raciti L, Nicoletti A, Zappia M. Clinical diagnostic tricks for detecting psychogenic gaze paralysis. Eur J Neurol. 2013; 20(8): e107-8.

17. Troost BT, Troost EG. Functional paralysis of horizontal gaze. Neurology. 1979; 29(1): 82-5.

18. Jankovic J. Diagnosis and treatment of psychogenic parkinsonism. J Neurol Neurosurg Psychiatry. 2011; 82(12): 1300-3.

19. Bronstein AM. Vision and vertigo: some visual aspects of vestibular disorders. J Neurol. 2004; 251(4): 381-7.

20. Zahn JR. Incidence and characteristics of voluntary nystagmus. J Neurol Neurosurg Psychiatry. 1978; 41(7): 617-23.

21. Bassani R. Images in clinical medicine. Voluntary nystagmus. The New England journal of medicine. 2012; 367(9): e13.

22. Lemos J, Eggenberger E. Saccadic intrusions: review and update. Curr Opin Neurol. 2013; 26(1): 59-66.

23. Meadows JC. Observations on a case of monocular diplopia of cerebral origin. J Neurol Sci. 1973; 18(2): 249-53.

24. Danchaivijitr C, Kennard C. Diplopia and eye movement disorders. J Neurol Neurosurg Psychiatry. 2004; 75 Suppl 4: iv24-31. 
25. Keane JR. Triplopia: thirteen patients from a neurology inpatient service. Arch Neurol. 2006; 63(3): 388-9.

26. Stone J. Pseudo-ptosis. Pract Neurol. 2002; 2: 364-5.

27. Devoize JL. "The other" Babinski's sign: paradoxical raising of the eyebrow in hemifacial spasm. J Neurol Neurosurg Psychiatry. 2001; 70(4): 516.

28. Stamey W, Jankovic J. The other Babinski sign in hemifacial spasm. Neurology. 2007; 69(4): 402-4.

29. Schwingenschuh P, Katschnig P, Edwards MJ, Teo JT, Korlipara LV, Rothwell JC, et al. The blink reflex recovery cycle differs between essential and presumed psychogenic blepharospasm. Neurology. 2011; 76(7): 610-4.

30. Tan EK, Jankovic J. Psychogenic hemifacial spasm. The Journal of neuropsychiatry and clinical neurosciences. 2001; 13(3): 380-4.

31. Gibson LM, Whiteley W. The differential diagnosis of suspected stroke: a systematic review. The journal of the Royal College of Physicians of Edinburgh. 2013; 43(2): 114-8.

32. Umapathi T, Venketasubramanian N, Leck KJ, Tan CB, Lee WL, Tjia H. Tongue deviation in acute ischaemic stroke: a study of supranuclear twelfth cranial nerve palsy in 300 stroke patients. Cerebrovasc Dis. 2000; 10(6): 462-5.

33. Yaltho TC, Jankovic J. The many faces of hemifacial spasm: differential diagnosis of unilateral facial spasms. Mov Disord. 2011; 26(9): 1582-92.

34. Baizabal-Carvallo JF, Fekete R. Recognizing uncommon presentations of psychogenic (functional) movement disorders. Tremor Other Hyperkinet Mov (N Y). 2015; 5: 279.

35. Margari F, Giannella G, Lecce PA, Fanizzi P, Toto M, Margari L. A childhood case of symptomatic essential and psychogenic palatal tremor. Neuropsychiatric disease and treatment. 2011; 7: 223-7.

36. Stamelou M, Saifee TA, Edwards MJ, Bhatia KP. Psychogenic palatal tremor may be underrecognized: reappraisal of a large series of cases. Mov Disord. 2012; 27(9): 1164-8.

37. Kim JS, Moon SY, Choi KD, Kim JH, Sharpe JA. Patterns of ocular oscillation in oculopalatal tremor: imaging correlations. Neurology. 2007; 68(14): 1128-35.

38. Lopez LI, Bronstein AM, Gresty MA, Du Boulay EP, Rudge P. Clinical and MRI correlates in 27 patients with acquired pendular nystagmus. Brain. 1996; 119 ( Pt 2): 465-72.

39. Baizabal-Carvallo JF, Jankovic J. The clinical features of psychogenic movement disorders resembling tics. J Neurol Neurosurg Psychiatry. 2014; 85(5): 573-5.

40. Demartini B, Ricciardi L, Parees I, Ganos C, Bhatia KP, Edwards MJ. A positive diagnosis of functional (psychogenic) tics. Eur J Neurol. 2014.

41. Demartini B, Ricciardi L, Parees I, Ganos C, Bhatia KP, Edwards MJ. A positive diagnosis of functional (psychogenic) tics. Eur J Neurol. 2015; 22(3): 527-e36.

42. Kolbrunner J, Menet AD, Seifert E. Psychogenic aphonia: no fixation even after a lengthy period of aphonia. Swiss medical weekly. 2010; 140(1-2): 12-7.

43. Parees I, Kojovic M, Pires C, Rubio-Agusti I, Saifee TA, Sadnicka A, et al. Physical precipitating factors in functional movement disorders. J Neurol Sci. 2014. 
44. Stone J, Reuber M, Carson A. Functional symptoms in neurology: mimics and chameleons. Pract Neurol. 2013; 13(2): 104-13.

45. Perez DL, Dworetzky BA, Dickerson BC, Leung L, Cohn R, Baslet G, et al. An integrative neurocircuit perspective on psychogenic nonepileptic seizures and functional movement disorders: neural functional unawareness. Clinical EEG and neuroscience. 2015; 46(1): 4-15.

46. Mehta AR, Rowe JB, Schrag AE. Imaging psychogenic movement disorders. Current neurology and neuroscience reports. 2013; 13(11): 402.

47. Schrag AE, Mehta AR, Bhatia KP, Brown RJ, Frackowiak RS, Trimble MR, et al. The functional neuroimaging correlates of psychogenic versus organic dystonia. Brain. 2013; 136(Pt 3): 770-81.

48. Aybek S, Nicholson TR, Zelaya F, O'Daly OG, Craig TJ, David AS, et al. Neural correlates of recall of life events in conversion disorder. JAMA psychiatry. 2014; 71(1): 52-60.

49. Nielsen G, Stone J, Edwards MJ. Physiotherapy for functional (psychogenic) motor symptoms: a systematic review. Journal of psychosomatic research. 2013; 75(2): 93-102.

50. Nielsen G, Ricciardi L, Demartini B, Hunter R, Joyce E, Edwards MJ. Outcomes of a 5-day physiotherapy programme for functional (psychogenic) motor disorders. J Neurol. 2015; 262(3): 674-81.

51. Jordbru AA, Smedstad LM, Klungsoyr O, Martinsen EW. Psychogenic gait disorder: a randomized controlled trial of physical rehabilitation with one-year follow-up. J Rehabil Med. 2014; 46(2): 181-7.

52. Demartini B, Batla A, Petrochilos P, Fisher L, Edwards MJ, Joyce E. Multidisciplinary treatment for functional neurological symptoms: a prospective study. J Neurol. 2014; 261(12): 2370-7.

53. LaFrance WC, Jr., Baird GL, Barry JJ, Blum AS, Frank Webb A, Keitner GI, et al. Multicenter pilot treatment trial for psychogenic nonepileptic seizures: a randomized clinical trial. JAMA psychiatry. 2014; 71(9): 997-1005.

54. Goldstein LH, Chalder T, Chigwedere C, Khondoker MR, Moriarty J, Toone BK, et al. Cognitive-behavioral therapy for psychogenic nonepileptic seizures: a pilot RCT. Neurology. 2010; 74(24): 1986-94.

55. Stone J, Carson A. Functional neurologic disorders. Continuum (Minneap Minn). 2015; 21(3 Behavioral Neurology and Neuropsychiatry): 818-37.

56. Peckham EL, Hallett M. Psychogenic movement disorders. Neurologic clinics. 2009; 27(3): 801-19, vii.

57. Stone J, Edwards M. Trick or treat? Showing patients with functional (psychogenic) motor symptoms their physical signs. Neurology. 2012; 79(3): 282-4.

58. Edwards MJ, Adams RA, Brown H, Parees I, Friston KJ. A Bayesian account of 'hysteria'. Brain. 2012.

59. Cogan DG, Freese CG, Jr. Spasm of the near reflex. AMA Arch Ophthalmol. 1955; 54(5): 752-9.

60. Christoff A, Christiansen SP. Spasm of the near reflex: treatment with miotics revisited. The American orthoptic journal. 2002; 52: 110-3.

61. Moore SS, L. Another approach to the treatment of accommodative spasm. . The American orthoptic journal. 1973; 23: 71-2. 
62. Pollak TA, Nicholson TR, Edwards MJ, David AS. A systematic review of transcranial magnetic stimulation in the treatment of functional (conversion) neurological symptoms. J Neurol Neurosurg Psychiatry. 2014; 85(2): 191-7.

63. Chastan N, Parain D. Psychogenic paralysis and recovery after motor cortex transcranial magnetic stimulation. Mov Disord. 2010; 25(10): 1501-4.

64. Schonfeldt-Lecuona C, Connemann BJ, Viviani R, Spitzer M, Herwig U. Transcranial magnetic stimulation in motor conversion disorder: a short case series. Journal of clinical neurophysiology : official publication of the American Electroencephalographic Society. 2006; 23(5): 472-5.

65. McWhirter L, Carson A, Stone J. The body electric: a long view of electrical therapy for functional neurological disorders. Brain. 2015; 138(Pt 4): 1113-20.

66. Shah BB, Chen R, Zurowski M, Kalia LV, Gunraj C, Lang AE. Repetitive transcranial magnetic stimulation plus standardized suggestion of benefit for functional movement disorders: an open label case series. Parkinsonism Relat Disord. 2015; 21(4): 407-12.

67. Garcin B, Roze E, Mesrati F, Cognat E, Fournier E, Vidailhet M, et al. Transcranial magnetic stimulation as an efficient treatment for psychogenic movement disorders. J Neurol Neurosurg Psychiatry. 2013; 84(9): 1043-6.

\section{Figure legends}

Figure 1. (A) Convergence spasm in a 28 year-old lady presenting with intermittent diplopia (see also Video 1). In this case, there is excessive and prolonged convergence in the left eye on attempted downgaze. (B) She is however able to fully abduct the left eye, ruling out an abducens nerve palsy. (C) Left-sided convergence spasm on downgaze. (D) Again, there if full abduction in the left eye, with miosis.

Figure 2. (A) Illustrative example of horizontal jerk nystagmus with a slow phase to the right (upward deflection), and a fast phase to the left (downward deflection). (B) Binocular horizontal electronystagmogram trace recorded from a patient with functional ("voluntary") nystagmus during convergence. Note the brief highfrequency oscillations seen during convergence lasting 1.5 seconds (see also Video 5). 
Figure 3 Functional facial movement disorder. (A) Left-sided platysma contraction with jaw deviation accompanied by a functional left hemiparesis. (B) Contraction of orbicularis. (C) Episodic bilateral facial spasm induced by testing eye movements sequential ptosis, platysma, and orbicularis contraction. (D) Right facial spasm induced by light giving the appearance of weakness (third picture). (E) Right platysma contraction with jaw deviation, giving the appearance of "mouth droop". (F) Persistent right unilateral facial spasm associated with jaw and tongue deviation. $(\mathrm{G})$ Episodic right eye and face contraction with upward movement of the mouth associated with hyperventilation.

\section{[TO BE RE-DRAWN]}

Figure 4. Position of eyelid and eyebrow in different types of facial spasm. (A) Blepharospasm. Bilateral (though may be asymmetric) eyelid closure. There may be bilateral raising of eyebrows on attempted eye opening. (B) "The other Babinski sign". Paradoxical raising of the eyebrow on the same side (arrow) as the eye closure in a patient with organic hemifacial spasm. (C) Functional orbicularis spasm with raised eyebrow on the opposite side to the eye closure (arrow).

Figure 5. Hemilingual spasm. (A) In an organic supranuclear hypoglossal palsy, the tongue deviates towards a lower facial weakness and limb weakness. (B) Medullary lesion with tongue deviation towards upper and lower facial weakness but away from limb weakness. There may be marked ipsilesional wasting of the tongue. (C) In a functional tongue spasm the tongue also deviates towards the side of the functional facial spasm. If functional limb weakness is present, it is usually on the same side as the facial spasm. 


\section{Video legends}

Video 1: Convergence spasm (1). The patient's eyelids are opened by the examiner to visualize the eye movements more clearly. The patient has a full range of horizontal movements in both eyes when looking right and left. On attempted downgaze there is a convergent eye movement in the left eye that persists on upgaze, improves transiently and partially when the eyes are brought to the primary position, and then deteriorates on further attempted downgaze. The convergence spasms remains during Dolls-head eye movements.

Video 2: Convergence spasm (2). The patient has a full range of horizontal eye movements. Eyelid flutter is seen during rightward gaze, and this increased on upgaze. During attempted downgaze, the right eye converges. There is marked eyelid flutter on sustained downgaze on both sides. The convergence improves during central gaze, and the patient is then able to abduct the eye fully, although she reports both double vision and blurring. The pupil constricts during the convergence spasm.

Video 3: Functional gaze limitation and effortful facial movements in the formal examination. The patient visibly struggles to make horizontal eye movements, often accompanied by eye closure and frowning. There appears to be a tubular field defect during the examination as she describes an inability to see the finger as it moves. A tubular defect was not observed when the testing visual fields to confrontation. We suspect the tubular defect shown in the video relates to the patient's belief that her 
inability to move her eyes also means that she would not see the finger that she is being asked to track (whereas it should remain in her peripheral vision).

Video 4: Normal eye movements during casual examination, becoming abnormal during the formal examination. As the patient describes her symptoms, she makes normal reflexive eye movements. When the eye movements are tested formally, she develops an effortful expression, accompanied by frequent eye closure, and poor task performance with the eyes making convoluted trajectories to reach the target. At one point her head falls forwards but she manages to regain her posture. Following the formal examination, her facial expression relaxes and she makes normal reflexive upward saccades.

Video 5: Functional nystagmus. Recurrent bursts of brief conjugate horizontal eye oscillations that occur during the formal examination with the patient looking straight ahead. Note that each episode lasts less than 2 seconds, although multiple episodes occur within the recording.

Video 6: Voluntary nystagmus demonstrated by one of the authors (AMB). Two brief episodes of rapid bursts of horizontal eye oscillation are shown. Each episode lasts 1.5 seconds during which there are high-frequency to-and-fro eye movements with no inter-saccadic interval. The eye movements were seen in both eyes, although for clarity only one eye is shown here.

Video 7: Right-sided facial spasm with platysma contraction and jaw deviation (also shown as Figure 3E). As the patient describes her symptoms, the orbicularis oris on 
the right has a tendency to contract and pull the mouth and cheek rightwards. On formal testing, there is more obvious contraction of the muscles of the right side of the mouth, extending to platysma. The contraction now persists beyond the examination, with more clear jaw deviation.

Video 8: Left sided facial spasm with platysma contraction and jaw deviation (also shown in Figure 3A).

Video 9: Right-sided facial spasm with bilateral orbicularis oculis and right-sided oris contraction (upward contraction of the mouth; also shown in Figure 3G). There is intermittent eye opening on the left, and intermittent raising of the eyebrow during eye opening. The facial spasm is variable during speech.

Video 10: Eye movement examination-induced facial spasm related to Figure 3 Case C. There is a normal range of vertical eye movements, but sustained convergence induced eyelid closure on the right, followed by gradual onset right-sided facial spasm with jaw deviation to the right, platysma contraction, and eventually involvement of both sides of the face. Eyelid closure improves during the evolution of the facial spasm, and there is intermittent head tremor associated with platysma contraction.

Video 11: Light-induced right-sided facial spasm (also shown in Figure 3D and Box 2). Contraction of the right orbicularis oris is intermittent following light exposure to the right eye, and varies in severity during speech.

Box 1 - Functional nystagmus 
A 17 year old man was preparing for his college examinations. Towards the end of the day, having been reading for several hours, he developed brief, rapid oscillation of the eyes leading to oscillopsia. The following day, fearing a neurological disease, they attended the GP, who referred the patient to the neurologist who arranged a normal MRI brain scan. Although reassured by the doctor, the patient continued to experience oscillopsia, limiting his ability to function. The symptoms settled when the patient received an explanation of the nature of the nystagmus.

\section{Box 2 (related to Video 10)}

A 40 year old woman described a 6 year history of recurrent attacks of right facial spasm often triggered by exposure to direct light or piercing noise. Each episode was characterised by abrupt onset of right-sided facial spasm, pain behind the right eye, photophobia, blurred vision and associated mild right hemiparesis, with altered sensation. These symptoms lasted between hours and weeks. Between episodes there were milder residual right-sided face, arm and leg symptoms, photophobia, low mood and fatigue. Light avoidance led to weeks without leaving the house.

She has low mood with hypersomnia, impaired memory and concentration, but not major depression or anxiety. Investigations included normal MRI of the brain, and cerebrospinal fluid (including negative oligoclonal bands).

On examination (see video 10) light in her right eye precipitated marked spasm on the right side of the face with variable contraction of orbicularis oculi and oris. This gives the appearance of right-sided weakness (but air escapes out of the left side of 
the mouth) with the tongue deviating to the left. There was persistent variable functional right arm and leg weakness with a positive hoovers sign.

She was diagnosed with recurrent functional facial spasm with associated functional limb weakness, photophobia and fatigue, partly related to migraine. 